\title{
La enseñanza del español en Francia en el siglo XVIII
}

Contexto, autores, obras y préstamos

\section{M. ${ }^{\text {a }}$ Carmen Cazorla Vivas}

\section{OpenEdition}

\section{Journals}

Edición electrónica

URL: https://journals.openedition.org/cher/2919

DOI: 10.4000/cher.2919

ISSN: 2803-5992

\section{Editor}

Presses universitaires de Strasbourg

\section{Edición impresa}

Fecha de publicación: 27 junio 2017

Paginación: 127-137

ISBN: 978-2-86820-961-0

ISSN: 1968-035X

\section{Referencia electrónica}

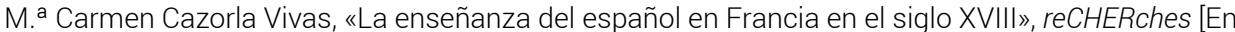
línea], 18 | 2017, Publicado el 01 diciembre 2021, consultado el 16 diciembre 2021. URL: http:// journals.openedition.org/cher/2919; DOI: https://doi.org/10.4000/cher.2919

\section{(c) (1) (9)}

Ce(tte) œuvre est mise à disposition selon les termes de la Licence Creative Commons Attribution -

Pas d'Utilisation Commerciale - Partage dans les Mêmes Conditions 4.0 International. 


\title{
La enseñanza del español en Francia en el siglo XVIII: contexto, autores, obras y préstamos'
}

\author{
M. ${ }^{a}$ Carmen Cazorla Vivas*
}

\begin{abstract}
G $\mathrm{n}$ el marco de las investigaciones sobre el siglo xviII, y más concretamente del español y el francés a través de sus diccionarios. Vamos a presentar de manera sintetizada algunos aspectos del contexto histórico-lingüístico en el que se desarrolla la enseñanza de estas dos lenguas, para mostrar a continuación un panorama de los principales autores y obras que jalonan esta época, prestando especial atención a aspectos como el lugar de publicación y el lugar de procedencia de estos lexicógrafos. Especialmente interesante resulta conocer la interrelación de los diccionarios bilingües con los monolingües de la época, ya que comparten una serie de características de su microestructura que nos permiten extraer la voz de unos en otros, y así nos aproximamos a la finalidad del Coloquio que nos ocupa estos días. Podremos ver esta influencia a través de muestras extraídas de algunos de los repertorios más conocidos de la época: concretamente del académico Diccionario de Autoridades (1726-1739) y de dos diccionarios bilingües español-francés: los de Séjournant (1759) y Cormon (1769).
\end{abstract}

\section{Contexto histórico-lingüístico alrededor del siglo XVIII}

Durante el siglo XVIII continuó, y aumentó, el estudio de lenguas modernas. A las razones políticas, diplomáticas y comerciales se fueron sumando necesidades

* Doctora en Filología hispánica y actualmente profesora en el Departamento de Lengua española de la Universidad Complutense de Madrid.

1 Este trabajo se enmarca en los trabajos llevados en el Proyecto Biblioteca Virtual de la Filología Española. Fase II. Consolidación, mejora y ampliación de los datos y de la web. Estudio de los materiales contenidos» (FFI2014-5381-P). Dicha biblioteca puede consultarse en http://biblioteca.ucm.es/nebrija 
docentes que ampliaron el número y tipo de usuarios y con el aumento de la demanda se necesitaban materiales para su aprendizaje, por lo que diccionarios, gramáticas o diálogos proliferaron².

En el caso de las dos lenguas que nos ocupan, todavía en esta centuria el español era más estudiado en Francia que al contrario, pero con el inicio de la supremacía francesa en Europa, su lengua se irá introduciendo en mayor medida. Concretamente, en España entra una dinastía francesa, cada vez más franceses vienen a España (tanto gente de la Corte como artistas, artesanos y obreros).

Las traducciones de libros franceses en España serán muy numerosas, y los manuales necesarios para la traducción y lectura de estos libros serán muy útiles y demandados. En las últimas décadas de la centuria se abordará la reforma de la enseñanza y en muchos centros educativos se introducirá el aprendizaje del francés.

Por lo que se refiere a la situación del español en Francia, en el siglo XVIII continúa el interés por aprenderlo: la colonia española en Francia era abundante, muchos de estos emigrados se ganaban la vida precisamente como profesores de español y todavía se mantenía el gran prestigio de la literatura española del Siglo de Oro, y las abundantes traducciones que se hicieron allí.

\section{Autores, lugares, obras}

\subsection{Procedencia de los autores}

La procedencia de los lexicógrafos autores de los diccionarios no es un tema menor. La cuestión de si un autor era español o francés, el nivel de dominio de ambas lenguas, dónde vivía, si se había ayudado de algún nativo de la otra lengua que no fuera la suya materna... Por ejemplo, en general, hasta el siglo XVIII la autoría solía ser única, basada en la propia competencia del redactor y, claro está, en los referentes lexicográficos que siguiera; pero en el siglo xIx se generalizó bastante el trabajo en parejas, con un autor español (o especializado en su enseñanza) y otro francés, y más tarde, sobre todo para los diccionarios enciclopédicos, el trabajo en equipo. Al menos así se reflejaba en las portadas e introducciones de las obras.

En el caso de la lexicografía español-francés del siglo xviII, los autores españoles serán mayoría en número, concretamente Francisco de la Torre y Ocón, con El maestro de las dos lenguas. Diccionario español y francés en dos tomos (Madrid, 1728-1731); Antonio María Herrero, Diccionario Universal francés y español más copioso que cuantos hasta ahora se han visto... (Madrid, Imprenta del Reyno, 1743-1744); Nicolás González de Mendoza, que escribió el Diccionario general de las dos lenguas española y francés (Madrid, Andrés

2 Hay muchos trabajos sobre la enseñanza de lengua en el siglo xviII. Algunos ellos, sin ánimo de exhaustividad, son Suárez Gómez (1956), Brunot (1967), Niederehe (1987), Verdonk (1991), Lépinette (1996), Kailuweit 1998), Sáez (2008) o Cazorla (2014). 
Ortega, 1761-1763) y el de Diego Antonio de Godoy, uno de los muchos jesuitas que tuvieron que abandonar España, compuso el Diccionario nuevo, portátil y manual, francés-español (Bolonia, Franceschi, 1798), y sobre todo, por el gran éxito e influencia que tuvo, Francisco Sobrino, con su Diccionario nuevo de las lenguas española y francesa (Bruselas, Foppens, 1705). El primer diccionario hispano-francés que abre la década está compuesto por Guillaume de Maunory, Grammaire et dictionnaire françois et español... (París, Vv. Claude Barbin, 1701), y tres de las obras más conocidas y reconocidas de la centuria estarán realizadas también por autores franceses: Pierre de Séjournant, Nouveau Dictionnaire Espagnol-François et Latin Composé Sur les Dictionnaires des Académies Royales de Madrid et de Paris (París, Jombert, 1759), François Cormon, Sobrino Aumentado o Nuevo Diccionario de las Lenguas Española, Francesa y Latina (Amberes, Hnos. de Tournes, 1769) y Claude-Marie Gattel (Lyon, 1790)³.

\subsection{Lugar de publicación}

Además de la procedencia de los autores, aspecto fundamental a la hora de encarar la redacción de una obra lexicográfica bilingüe, hay otras cuestiones igualmente interesantes, como el lugar de publicación. A lo largo del siglo XVIII, los Países Bajos siguen siendo un lugar de impresión puntero, y en ciudades como Bruselas o Amberes siguen apareciendo algunas de las obras que más éxito tendrán, como son las de Sobrino (Bruselas) y Cormon (Amberes). París, por lo que se refiere a la lexicografía bilingüe hispano-francesa, empezará a despuntar también y otro de los diccionarios de referencia, el de Séjournant, se publicará en esta ciudad, así como el de Maunory, que es el que inicia la centuria (aunque en su caso no será tan conocido ni difundido). Otra ciudad francesa, Lyon, venía siendo igualmente un lugar prestigioso por lo que concierne a la impresión y allí salió el repertorio de Gattel, que era natural de esta ciudad. Por lo que concierne a Madrid, tres de los repertorios mencionados (De la Torre, Herrero y González de Mendoza), se publicaron allí, pero en ninguno de los tres casos fueron obras que tuvieran especial difusión; de hecho, ninguna de ellas conoció una segunda edición, mientras que las de Sobrino, Séjournant, Cormon o Gattel se editaron en numerosas ocasiones y significaron una referencia para los autores posteriores. Durante el siglo XVIII, las obras que se imprimieron en Madrid no fueron las que irradiaron más expectación. Esta situación irá modificándose en cierta manera durante el siglo XIX, y aunque entonces París sigue siendo un centro difusor fundamental, desde Madrid llegarán repertorios que sí se contarán entre los más importantes de esa centuria, como los de A. Capmany, R. J. Domínguez o D. Gildo.

3 Sobre la lexicografía bilingüe español-francés pueden verse, entre otros, Alvar Ezquerra (2004 y 2007); Bruña Cuevas (2006, 2008a y 2008b); Cazorla (2002a, 2002b, 2002c, 2002d, 2002-2004, 2008a, 2012); García Bascuñana (1996); von Gemmingen (2001). 


\subsection{Maestros de lenguas}

Otro aspecto destacado entre las características de los autores de diccionarios en el xviII es que muchos de ellos son 'maestros de lenguas', en el sentido de que publican no solo repertorios lexicográficos español-francés, sino también con otros idiomas o monolingües, gramáticas o diálogos; por ejemplo, Gattel, autor de diccionarios español-inglés, monolingüe francés, gramática italiana; Maunory escribe y publica conjuntamente su diccionario y una gramática del francés; de la Torre, también con métodos gramaticales y de pronunciación o Francisco Sobrino, autor de gramáticas, diálogos y cartas (sobre los Diálogos de Sobrino: Sáez 2002).

\section{Itinerarios y préstamos: la voz del otro en un diccionario}

En todas las épocas y todas las lenguas, los diccionarios que van apareciendo no surgen de la nada, sino que siguen, en mayor o menor medida, las líneas que van marcando sus predecesores. Un lexicógrafo no empieza a trabajar en una hoja en blanco, sino que tiene a su disposición las obras que lo han precedido. La forma de trabajo en la lexicografía bilingüe no es diferente, y además, durante el siglo xviII no se puede hacer un estudio a fondo de la lexicografía bilingüe de la época sin tener presente la lexicografía monolingüe, que será base y modelo de muchos de los repertorios bilingües. En el caso del español, los diccionarios académicos, desde el primero de Autoridades (1726-1739), estarán presentes de manera evidente y continua, y para el francés, también su repertorio académico será referencia (Dictionnaire de l'Académie française, 1694), aunque no de manera tan destacada como su equivalente español. Así, encontramos la voz de un autor en otros posteriores que lo toman como modelo; unas veces esta presencia es más que evidente, por el gran parecido que se da en muchas definiciones, y en otras ocasiones es más difícil encontrar el modelo seguido.

Los diccionarios monolingües y bilingües de estos siglos comparten una serie de características que los acercan y que permiten cotejarlos entre ellos. Desde nuestra perspectiva actual del siglo xxI, un repertorio monolingüe $\mathrm{y}$ otro bilingüe son obras con un contenido y una estructura totalmente diferente, pero a lo largo del siglo XVIII los diccionarios bilingües contienen, por ejemplo, amplias definiciones, similares a las que encontramos en un repertorio monolingüe, solo que se ofrecen en más de una lengua; es decir, no se limitan a la inclusión de uno o varios equivalentes, como es lo habitual en la actualidad. Muchas características hacen que estos dos tipos de diccionarios estén más cercanos de lo que podemos pensar desde nuestra perspectiva actual.

Para mostrar la presencia del repertorio académico en diccionarios bilingües, y de un diccionario bilingüe en otro posterior, incluimos a continuación unas muestras tomadas de tres diccionarios:

- Real Academia Española, Diccionario de Autoridades, Madrid, 1726-1739

- Pierre de Séjournant, Nouveau Dictionnaire Espagnol-François et Latin..., Paris, Jombert, 1759 
- François Cormon, Sobrino Aumentado o Nuevo Diccionario de las Lenguas Española, Francesa y Latina, Amberes, Hermanos de Tournes, 1769

(Autoridades):

ABESANA, s. f. Yunta de bueyes de labor. Término usado en Andalucía, donde llaman assí a las yuntas, o pares de bueyes, que son de un dueño, y aran en una misma parte. Lat. [...]

(Séjournant) :

ABESANA, s. f. Terme du royaume d'Andalousie; attelage de boeufs de labour.

(Cormon):

ABESANA, s.f. Terme du Royaume d'Andalousie. Couple de boeufs qui appartiennent au même maître, et qui labourent le même champ. Lat. Bonum jugum trahentium copia.

(Autoridades)

ALEGRARSE, Además del sentido literal, en el Dialecto antiguo forense de Aragón, vale Aprovecharse, alegar algo en defensa propria, para eximirse de culpa, y de los cargos que se le oponen y hacen.

(Séjournant)

ALEGRARSE, Se réjouir, s'égaier, [...] Et en terme de pratique ancienne du royaume d'Aragon, profiter de l'occasion, se couvrir d'un privilège, l'alléguer en sa défense pour s'exempter de la peine meritée.

(Cormon)

ALEGRARSE, Se réjouir, outre le sens littéral, dans le stile du Droit Aragonnois, signifie alléguer quelque chose pour sa défense [...]

\section{(Autoridades)}

Adula. S. f. Término, o partido, y principalmente se llama assi el que no tiene regador destinado. Viene del Griego Adoulos, que significa el que no tiene siervos, o criados. Voz usada en Aragón. Lat. Locus rigationibus carens. [...]

(Séjounant)

ADULA, s.f., T. du Royaume d'Aragon. Territoire qu'on n'arrose pas, faute d'avoir un homme destiné pour y conduire l'eau. Lat. Locus irrigationibus carens.

\section{(Cormon)}

${ }^{*}$ ADULA, s.f., Terme Aragonois. Territoire qu'on n'arrose pas, faute de domestique pour y conduire l'eau. Lat. Locus irrigationibus carens.

En el caso de los artículos Abesana, Adula y Alegrarse podemos observar cómo las definiciones en los tres diccionarios son parecidas y en los tres casos marcadas diatópicamente, y representan voces que no son de uso muy común y sin embargo, aparecen en diccionarios bilingües precisamente porque su modelo es el repertorio académico, en el que abundan voces marcadas de esta forma, especialmente del Reino de Aragón (Cazorla 2008b, 2009). 
(Autoridades)

ALGARRADA, Se llama también en la Corte el acto de conducir los toros por la mañana temprano, para encerrarlos en los toriles que están en la Plaza [...].

(Séjournant)

ALGARRADA, Conduite ou entreé des toreaux dans Madrid, pour la grande course dans les réjouissances publiques $[\ldots]$

(Cormon)

ALGARRADA. On appelle ainsi à Madrid la conduite qu'on fait le matin des taureaux, pour les enfermer dans les loges $[\ldots]$

En el caso del artículo Algarrada, observamos que es una voz sumamente específica, que se refiere a una costumbre muy concreta del pueblo de la corte de Madrid (y así aparece marcada) y sin embargo la encontramos también en Séjournant y Cormon, con definiciones similares, especialmente la de este último. De nuevo siguen el modelo académico.

\section{(Autoridades)}

ACETRE. Covarr. dice que también se llama assi el hyssopo con que se esparce el agua bendita, y que por la semejanza que tiene con el cetro se pudo llamar acetre. Es voz que no tiene uso. Lat. Aspersorium. Hyssopus.

$[\ldots]$

ACETRE, Se llama en el Reino de Granada y en otras partes la calderilla o caldero pequeño, con que se saca agua de las tinajas o pozos, que ordinariamente es de cobre.

\section{(Séjournant)}

ACETRE, Se dit aussi du Goupillon, selon Covarrubias; mais il est hors d'usage. Lat. Aspersorium, ii.

[...]

ACETRE, Est aussi un petit chaudron à bec qui sert à puiser de l'eau d'un puits ou d'une tinaja. Lat. Situla, ae.

(Cormon)

ACETRE. On appelle ainsi dans le Royaume de Grenade, un petit chaudron de cuivre à bec avec lequel on tire l'eau d'un puits.

Y en el caso del artículo Acetre de nuevo se observa la influencia de un repertorio en los otros, si bien ahora hallamos otra voz en el interior, la referencia del repertorio académico a Covarrubias, que se mantiene en Séjournant, pero no en Cormon; y sin embargo, en otra de las acepciones, que aparece marcada diatópicamente como del reino de Granada, Séjournant ofrece una definición tomada claramente de Autoridades, pero eliminando la indicación regional, que Cormon sí mantiene.

Y el siguiente artículo, Aciguatado, nos muestra de nuevo este triple camino:

\section{(Autoridades)}

ACIGUATADO,DA, adj. Voz Mexicana, introducida y mui usada en Andalucía, que equivale a Atiriciado, pálido y de color amarillo. [...] 
(Séjournant)

ACIGUATADO,DA: adj. Terme Mexicain, introduit en Andalousie, qui se dit de ceux qui ont une espèce de jaunisse, qui sont blêmes pâles.

(Cormon)

ACIGUATADO, DA, [Terme Mexicain en usage dans l'Andalousie]. Jaune, pâle, défait.

Y en los siguientes artículos, Adjuntos, Ado las dan las toman, son voces no marcadas diatópicamente en las que encontramos de nuevo el camino trazado entre los repertorios :

(Autoridades)

Adjuntos. Se dicen los que se dan por agregados, o compañeros para el conocimiento de alguna causa : y especialmente llaman assi los que en los Cabildos de muchas Iglesias Cathedrales de España se nombran por los mismos Cabildos, por Bula y privilegio que tienen dichas Iglesias, para conocer juntos con el Obispo, y determinar sobre las causas criminales de los Capitulares de las mismas Iglesias. Lat. Judices adjuncti.

(Séjournant)

ADJUNTOS. Adjoints, nom que l'on donne à ceux qui se joignent ensemble par commission particulière, ou par privilège, pour connoître, juger d'une affaire civile ou criminelle, \& spécialement dans les Chapitres d'Églises Cathédrales, losqu'il y a quelques plaintes contre un des Chanoines ou autres dépendans desdits Chapitres.

(Cormon)

ADJUNTOS. Adjoints, nom que l'on donne à ceux qui se joignent ensemble par commission particulière ou par privilège, pour connoître, juger d'une affaire civile ou criminelle, spécialement dans les chapitres d'Églises Cathédrales, losqu'il y a quelque plainte contre un des Chanoines, ou autres personnes qui dépendent des dits Chapitres.

\section{(Autoridades)}

Ado las dan, las toman. Refr. Que da a entender, que en donde se logra alguna ganancia, también se halla alguna pérdida o riesgo: y que quien se atreve a decir una mala palabra, o hacer alguna mala obra, debe esperar y temer le suceda otro tanto. Lat. Haud incruenta victoria.

(Séjournant)

No está recogido este refrán.

\section{(Cormon)}

Refr. Ado las dan, las toman. Ce proverbe signifie que là où l'on gagne des batailles, on les perd aussi, \& que ceux qui parlent, ou qui agissent mal, doivent s'attendre à la même chose.

\section{Conclusiones}

Al acercarnos a la investigación lingüística sobre el siglo XVIII podemos abordarla desde diferentes perspectivas. Por nuestra parte, nos interesa 
especialmente el estudio de lenguas modernas en esta época, y más concretamente del español y el francés. Para realizar un análisis completo y pormenorizado de la enseñanza y aprendizaje de lenguas en el siglo xvin hay que tener en cuenta diversos aspectos, como el contexto histórico-lingüístico de la época, cuáles eran las motivaciones que impulsaban el mayor o menor estudio de una lengua en un país, algunas cuestiones relacionadas con los autores, como su lengua materna, su profesión, en qué centros de impresión se publicaron las diferentes obras y, por supuesto, el contenido de los materiales que se usaban en el aprendizaje de lenguas: gramáticas, diálogos, diccionarios, cartas...

En el presente trabajo nos hemos acercado a diccionarios bilingües que se publicaban y se empleaban en el estudio de lenguas. A lo largo del siglo xVIII encontramos una serie de repertorios lexicográficos que presentan características destacadas, especialmente una microestructura amplia, con definiciones extensas, a la manera de las que encontramos habitualmente en los diccionarios monolingües, y es que estos serán, aunque desde nuestra perspectiva actual pueda resultar extraño, referencia clara de los repertorios bilingües. Los diccionarios académicos, el francés y el español, pero sobre todo este último, se presenta como modelo de muchas de las obras bilingües publicadas. Presentamos una muestra de artículos que nos enseñan esta influencia, esta 'voz del otro' que tanto ha guiado el encuentro que sirve de marco a los trabajos incluidos en este volumen.

Nos parece interesante concluir contribuyendo a la reflexión y la apertura de vías de investigación lexicográfica, con la relación de la lexicografía monolingüe $\mathrm{y}$ la bilingüe, que en el siglo xx siguen caminos paralelos, pero que en centurias anteriores muestran una interrelación sumamente interesante y que permiten variadas líneas de investigación y contraste.

\section{Bibliografía}

Fuentes primarias

Académie Française, 1694, Dictionnaire de l'Académie Françoise, Paris, Veuve Jean Baptiste Coignard.

Cormon F., 1769, Sobrino Aumentado o Nuevo Diccionario de las Lenguas Española, Francesa y Latina, Amberes, Hermanos de Tournes.

Covarrubias S. de, 1611, Tesoro de la Lengua Castellana o Española, Madrid.

Gattel C.M., 1790, Nouveau Dictionnaire Espagnol et François, François et Espagnol, avec l'Interpretation Latine de chaque mot, Lyon, Bruyset Frères.

Godoy D. A., 1795, Diccionario nuevo portátil y manual francés-español, Bolonia, Gaspar Franceschi.

González de Mendoza N., 1761-1763, Diccionario general de las dos lenguas española y francesa. esta primera parte será de español en francés y la segunda de francés en español, Madrid, Andrés Ortega. 
Herrero A. M., 1743-1744, Diccionario Universal francés y español más copioso que cuantos hasta ahora se han visto, el qual contiene todos los términos usados en la lengua francesa, con las frasses, y locuciones proprias, y figuradas de todos estilos y refranes y todo lo necessario para la perfecta inteligencia de dicho idioma, compuesta por El Dr. D. Antonio María Herrero, Madrid, Imprenta del Reyno.

Maunory G. (de), 1701, Grammaire et dictionnaire françois et español, nouvellement composez par monsieur [Guillaume] De Maunory, suivant l'usage de la cour d'espagne, Paris, Vve. Claude Barbin.

Real Academia Española, 1726-1739, Diccionario de Autoridades, Madrid (Ed. Facsímil, Madrid, Gredos, 1963).

Séjournant P. (de), 1759, Nouveau Dictionnaire Espagnol-François et Latin Composé Sur les Dictionnaires des Académies Royales de Madrid et de Paris, Paris, Jombert.

Sobrino F., 1705, Diccionario nuevo de las dos lenguas francesa-española $y$ francesa-española, Bruselas, François Foppens.

Torre y Ocón F. (de la), 1728-1731, El maestro de las dos lenguas. Diccionario español y francés en dos tomos, Madrid, Imprenta de Juan de Aritzia.

Estudios

Alvar Ezquerra M., 2004, «Presencias de la lexicografía francesa en la española», in: M. ${ }^{a}$ V. Galloso y J. Prado (eds.), Huelva, Servicio de Publicaciones de la Universidad, p. 23-33

Alvar Ezquerra M., 2007, «Panorama de la lexicografía del español en el siglo XVIII», in: Historiografía de la Lingüística en el ámbito hispánico. Fundamentos epistemológicos y metodológicos, Madrid, Arco/Libros, p. 269-327.

Brunot F., 1967, «Le français en Espagne», in: Histoire de la langue française des origines à 1900, VIII, Paris, Colin, p. 39-75.

Bruña Cuevas M., 2006, «El Diccionario universal francés y español (1744) de Antonio María Herrero», in: M. Bruña, M. ${ }^{a}$ G. Caballos (eds.), La cultura del otro: español en Francia y francés en España, Sevilla, APFUE, p. 133-147.

Bruña Cuevas M., 2008a, «La producción lexicográfica con el francés y el español durante los siglos XVI a XIX ", Philologia Hispalensis, 22, p. 37-111.

Bruña Cuevas M., 2008b, «El lugar de edición de los diccionarios francés-español (siglos Xvi a Xxi)», in: M. Campos, E. M. ${ }^{a}$ González y J. I. Pérez Pascual (eds.), La lexicografía bilingüe y didáctica: ayer y hoy. Anexos Revista de Lexicografía, 8, A Coruña, Universidade da Coruña, p. 9-20

Cazorla Vivas M. ${ }^{a}$ del C., 2002a, Lexicografía bilingüe con el español y el francés de los siglos XVIII y XIX, Madrid, Universidad Complutense-Colección Tesis Digital, http://www.ucm.es/BUCM/tesis/fll/ucm-t26053.pdf 
Cazorla Vivas M. ${ }^{a}$ del C., 2002b, «Panorama de la lexicografía hispano-francesa del siglo viII: el Diccionario nuevo portátil y manual francés-español de Diego Antonio de Godoy (1795)», Res Diachronicae, I, p. 115-123.

Cazorla Vivas M. ${ }^{a}$ del C., 2002c, «Una aportación más a la lexicografía bilingüe del siglo xvin el Nouveau dictionnaire espagnol, français et latin de Pierre de Séjournant (1759)», in: A. Bernabé, J. A. Berenguer, M. Cantarero y J.C. de Torres (eds.), Presente y futuro de la Lingüística en España, Madrid, SEL, II, p. 353-361.

Cazorla Vivas M. a del C., 2002d, «Una incursión en lexicografía bilingüe del siglo XVIII: la obra de Francisco Sobrino», in: M. ${ }^{a}$ D. Muñoz, A. I. Rodríguez et al., Actas del IV Congreso de Lingüística General, Cádiz, Universidad de CádizUniversidad de Alcalá, p. 269-306.

Cazorla Vivas M. ${ }^{a}$ del C., 2002-2004, «El Diccionario Universal Francés-español de Herrero y Rubira (1744)», Anuario de Filología Aragonesa, LIX-LX, p. 301-323.

Cazorla Vivas M. ${ }^{a}$ del C., 2008a, «Diccionarios en el Siglo de las Luces: el repertorio bilingue de N. González de Mendoza (1761-1763)», en M. Campos, E. M. ${ }^{a}$ González y J. I. Pérez Pascual (eds.), La lexicografía bilingüe y didáctica: ayer y hoy. Anexos Revista de Lexicografía, 8, A Coruña, Universidade da Coruña, p. 29-40.

Cazorla Vivas M. ${ }^{a}$ del C., 2008b, «Échantillon de répérage de spécialité dans la lexicographie bilingue du XviII ${ }^{\mathrm{e}}$ siècle: F. Sobrino et ses sources», Cahiers de lexicologie, 93-2, p. 5-26.

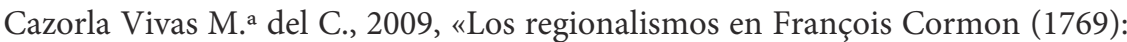
tras los pasos del Diccionario de Autoridades", Revista de Filología de La Laguna, 27, p. 53-73.

Cazorla Vivas M. ${ }^{a}$ del C., 2012, «Traductores y maestros de lenguas: gramáticas y vocabularios, el caso de De la Torre y Ocón (1728-1731)», Cuadernos dieciochistas, 13, p. 51-73.

Cazorla Vivas M. ${ }^{\text {a }}$ del C., 2014, Diccionarios y estudio de lenguas modernas en el Siglo de las Luces. Tradición y revolución lexicográfica en el Siglo de las Luces, Madrid, Liceus Ediciones.

García Bascuñana J. F., 1996, «Contribución al estudio de los diccionarios bilingües francés-español/español-francés: aproximación histórica y metodológica», in: E. Forgas (coord.), Léxico y Diccionarios, Universidad Rovira y Virgili, Valls, p. 91-103.

Gemmingen B. (von), 2001, «Estudios sobre el Diccionario español-Francés, Francés-Español del Abate Marie-Claude Gattel (1790)», in: A. M. Medina Guerra (coord.), Lexicografía española, Barcelona, Ariel, p. 211-242.

Kailuweit R., 1998, «Lengua y política en el s. XVIII - El español frente al francés», in: C. García Turza, F. González Bachiller y J. Mangado Martínez, Actas del IV Congreso AHLE, Madrid, Arco/Libros, p. 497-506. 
Lépinette B., 1996, «Contribution à l'histoire du français hors de France. Caractéristiques culturelles et sociales des maîtres et de leur enseignement en Espagne», in : J. F. García Bascuñana, B. Lépinette y C. Roig (éd.), Documents pour l'histoire du français et sa présence dans la Péninsule Ibérique. Actes du colloque de la SIHFLES tenu à Tarragone (Université Rovira i Virgili) du 28 au 30 septembre 1995, décembre, n 18, p. 189-212.

Niederehe H.J., 1987, «Les dictionnaires franco-espagnols jusqu'en 1800», Histoire, Épistemologie, Langage, IX-2, p. 13-26.

Sáez Rivera D., 2002, "Vida y obra de Francisco Sobrino (con breves noticias sobre Félix Antonio de Alvarado y Fray Gerónimo de Gracián», de los Diálogos nuevos (1708) de Francisco Sobrino, Anexos de la Revista Lemir, http://parnaseo.uv.es/Lemir/Textos/Sobrino/INTRO.pdf. Fecha de consulta: septiembre de 2013.

Sáez Rivera D., 2008, La lengua de las gramáticas y métodos de español como lengua extranjera en Europa (1640-1726), tesis doctoral, E-Prints http:// eprints.ucm.es/7813.

Suárez Gómez G., 1956, La enseñanza del francés en españa (Comentario a una bibliografía hasta 1850): tesis inédita, Madrid. Publicada recientemente, con una edición realizada por J. F. García Bascuñana y E. Juan Oliva, Barcelona, PPU, 2008.

Verdonk R., 1991, «La lexicographie bilingue espagnol-français, françaisespagnol», in: F.J. Haussmann et al., Wörterbücher: ein internationales Handbuch zur Lexicographie. Dictionaries, vol. III, Berlin, Walter de Gruyter, p. 2976-2987. 